\title{
Different Brain Circuits Underlie Motor and Perceptual Representations of Temporal Intervals
}

\author{
Domenica Bueti, Vincent Walsh, Chris Frith, and Geraint Rees
}

\begin{abstract}
In everyday life, temporal information is used for both perception and action, but whether these two functions reflect the operation of similar or different neural circuits is unclear. We used functional magnetic resonance imaging to investigate the neural correlates of processing temporal information when either a motor or a perceptual representation is used. Participants viewed two identical sequences of visual stimuli and used the information differently to perform either a temporal reproduction or a temporal estimation task. By comparing brain activity evoked by these tasks and control conditions, we explored commonalities and differences in brain areas involved in reproduction and estimation of temporal intervals. The basal ganglia and the cerebellum were commonly active in both
\end{abstract}

\section{INTRODUCTION}

Precise timing is essential for many human behaviors. Reaching for an object requires a specific temporal pattern of activity among the muscles of the shoulder, arm, and wrist. Timing information is also used for purely perceptual purposes. For example, predicting when a traffic light will change to red demands an accurate estimate of relevant durations, such as how long the light has been yellow. These examples emphasize that temporal information is used for both action and perception; and that in everyday life, temporal intervals must be both perceived and produced correctly. However, whether similar or different brain circuits are responsible for using time in both perception and action remains unresolved. Purely behavioral studies suggest that perceiving durations and acting in time may require shared pacemakers. For example, presentation of a rapid series of auditory or visual stimuli systematically increases or decreases perceived or produced time intervals (Treisman, Faulkner, \& Naish, 1992; Treisman, Faulkner, Naish, \& Brogan, 1990). Moreover, increases in performance variability as the duration of a stimulus increases are comparable for tasks requiring both discrimination and production of time intervals (Ivry \& Hazeltine, 1995; Keele, Pokorny, Corcos, \& Ivry, 1985).

University College London, UK temporal tasks, consistent with suggestions that perception and production of time are subserved by the same mechanisms. However, only in the reproduction task was activity observed in a wider cortical network including the right preSMA, left middle frontal gyrus, left premotor cortex, with a more reliable activity in the right inferior parietal cortex, left fusiform gyrus, and the right extrastriate visual area V5/MT. Our findings point to a role for the parietal cortex as an interface between sensory and motor processes and suggest that it may be a key node in translation of temporal information into action. Furthermore, we discuss the potential importance of the extrastriate cortex in processing visual time in the context of recent findings.

Furthermore, patients with cerebellar lesions and Parkinson's disease are impaired in both motor and perceptual time tasks (O'Boyle, Freeman, \& Cody, 1996; Ivry \& Keele, 1989). Although not always in agreement, studies of timing in patients with Parkinson's disease (Harrington, Haaland, \& Hermanowicz, 1998; Artieda, Pastor, Lacruz, \& Obeso, 1992) and cerebellar lesions (Spencer, Zelaznik, Diedrichsen, \& Ivry, 2003; Ivry, Keele, \& Diener, 1988), together with pharmacological investigations in animals (Matell, Meck, \& Nicolelis, 2003; Maricq \& Church, 1983) as well as electrophysiological (Schubotz \& Friederici, 1997) and neuroimaging studies in healthy humans (see Coull, Vidal, Nazarian, \& Macar, 2004; Nenadic et al., 2003; Lewis \& Miall, 2003a, for a review), are often interpreted as reflecting common involvement of the cerebellum and the basal ganglia in timekeeping operations. Taken together, these data suggest a common subcortical network of timing-related areas underpinning the use of time for both action and perception.

The functional role for cortical areas in motor and perceptual timing is less explored and more controversial than that of subcortical areas. Nevertheless, an increasing number of imaging studies using different tasks (finger tapping vs. perceptual discrimination) and different range of durations (milliseconds to seconds) identify the supplementary motor area (SMA), the premotor, the dorsolateral prefrontal, and the inferior 
parietal cortices as structures generically implicated in time processing (Coull et al., 2004; Rao, Mayer, \& Harrington, 2001; Schubotz, Friederici, \& von Cramon, 2000). However, none of those areas seem to be exclusively linked to motor rather than perceptual time processing or vice versa.

It has been suggested that different mechanisms exist for the measurement of different temporal durations (Gibbon, Malapani, Dale, \& Gallistel, 1997), for automatic and cognitive form of timing (Lewis \& Miall, 2003a), for motor versus nonmotor timing (Clarke, Ivry, Grinband, \& Shimizu, 1996), and for timing of continuous versus discontinuous movement (Spencer et al., 2003; Rammsayer, 1999). However, until now there have been no studies that directly compared the processing of temporal information for perceptual and motor purposes. In recent purely behavioral work (Bueti \& Walsh, submitted), we have established the generality of the perception/action distinction in the domain of timing. In that study, we asked subjects either to reproduce or estimate durations (ranging from 300 to $1200 \mathrm{msec}$ ) after a short or long delay period (1-8 sec). Although in the two tasks the motor and the perceptual components were not totally dissociated, we found significant differences in behavior between tasks. Increasing temporal delay between the presentation of the standard duration and the response of each subject significantly increased their bias to over/underestimation in the reproduction but not in the estimation task. Motivated by these behavioral findings, we sought to investigate whether there was also a dissociation in the neural mechanisms underlying this putative perception/action distinction.

We used the same task design, employing a time reproduction and a time estimation task while measuring brain activity using high-field functional magnetic resonance imaging (fMRI). In the time reproduction ("action") task (A), subjects were required to make a motor response to initiate and terminate a temporal interval, reproducing a standard duration with a timed action. In contrast, in the time estimation ("perception") task (P), subjects were required to make a motor response only when they had perceptually judged a visual stimulus to have been displayed for a time interval as close as possible to the standard. Both of these tasks required some motor preparation, a motor response; and in both tasks there was always a visual presentation of the time interval. The only difference between them is that the estimation of the whole interval is operationalized by a motor act in task A, whereas it is based entirely on a perceptual judgment in task P. These two tasks were directly comparable as they shared common encoding and storing of temporal information, but differed in how subjects used time information to make responses (timed movement vs. perceptual judgment). In this way, we sought to replicate our earlier behavioral findings, but now to test the new hypothesis that two different neural circuits underlie differences in encoding time using a motor or a perceptual representation.

\section{METHODS}

\section{Subjects}

Fourteen healthy volunteers (10 women, aged 19-35 years, mean $=25$ years, $S D=5.2$ years) gave written informed consent to participate in the study, which was approved by the Institute of Neurology and the National Hospital for Neurology and Neurosurgery Joint Ethics Committee.

\section{Stimuli}

There were four different experimental conditions: an action condition (A), a perception condition (P), and two corresponding matched control conditions (Control for Action [CA] and Control for Perception [CP]; see Figure 1). For every condition, identical visual stimuli were presented to participants on each trial; conditions differed in how that visual information was to be used by the participants. A red square (subtending $2^{\circ}$ of visual angle) was presented at fixation for one of four possible standard durations (300, 600, 900, or $1200 \mathrm{msec}$ ). The square was then replaced by a fixation cross for a variable delay period ( 1 or $8 \mathrm{sec}$ ). During this delay period, participants were instructed to hold either (A and $\mathrm{P}$ conditions) or ignore (CA and $\mathrm{CP}$ ) temporal information. After the delay period, in Condition A the visual presentation at fixation of the word "now" instructed participants to press and hold down a key to reproduce the length of the standard duration. Pressing down the key led to the appearance of a second identical red square that disappeared when the key was released. After the delay period in task $\mathrm{P}$, the appearance of a second, identical red square instructed subjects to press a key when the duration of this second red square matched the duration of the standard. Pressing this key led to the disappearance of the second red square. Thus, in the "action" (A) task, the subject's keypress began and ended the temporal duration. In other words, the estimation of the whole interval was begun and ended by a motor act. In the "perception" (P) task, the subject had no control over the onset of the interval, and the beginning of the temporal estimation must, therefore, be made based on an external cue (the second square on the computer display). In the A task, our intention was to make subjects judge the interval based on the time for which they had pressed, using a motor representation. In contrast, in the $\mathrm{P}$ task, our intention was to make subjects judge the interval based on the time for which the square had been displayed on the screen, using a perceptual representation. The two tasks were thus well matched for visual stimulation and for information to be stored and recalled after the delay period. 
Figure 1. Sequence of events in each trial for the four tasks. The first part of the trial was the same in each task. First, a red square was displayed on the screen for a standard duration and was followed by a blank interval. After the delay in task A, subjects were required to press and hold a button to reproduce the standard duration. Pressing the button led to the appearance of a second red square identical to the first. In task P, subjects were instructed to interrupt the presentation of a second, identical red square when its duration matched the standard. In control task CA, subjects pressed a key when the second square appeared and released it when it disappeared. In control task $\mathrm{CP}$, subjects pressed the key when the second square disappeared.

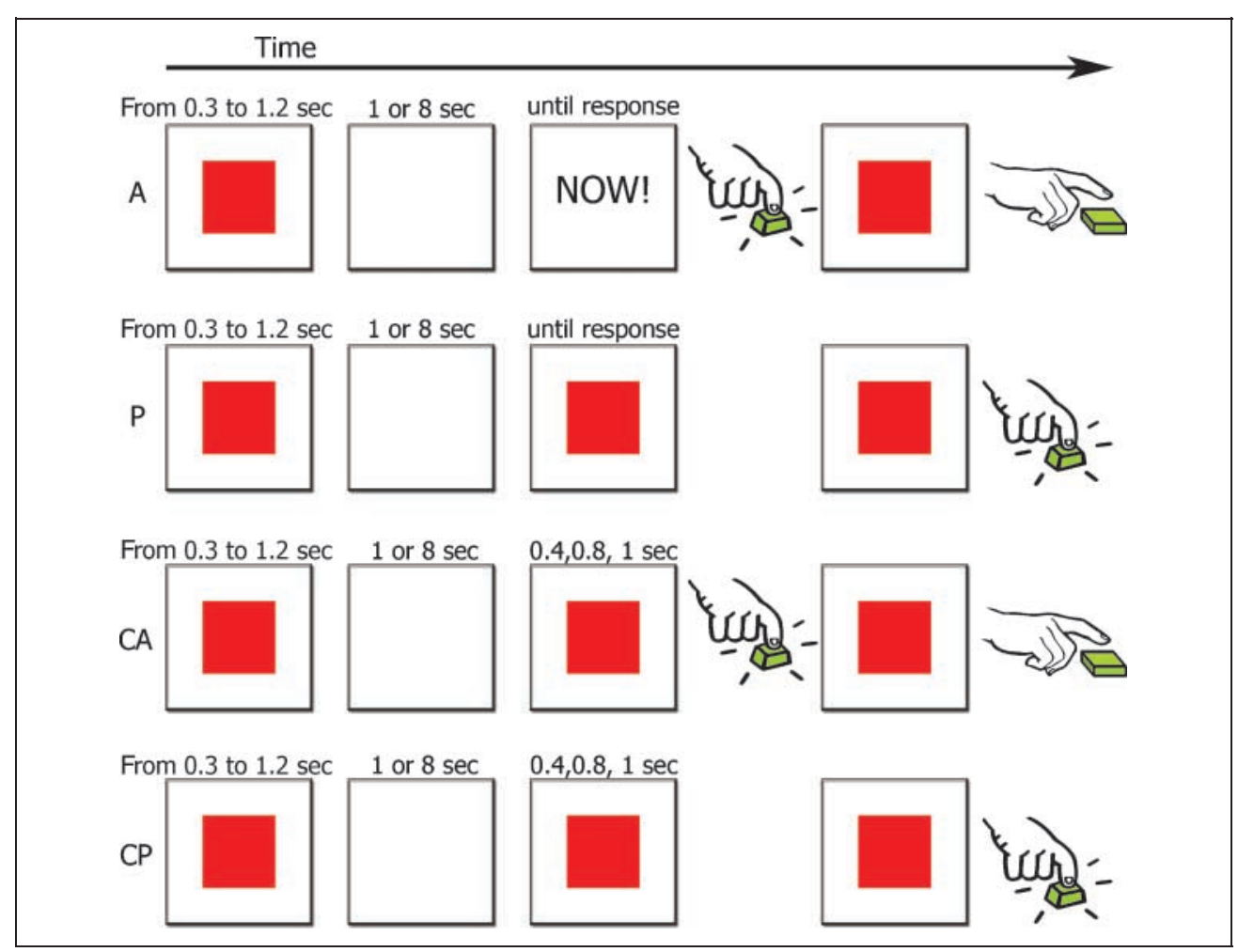

Two matched control conditions were also employed. After the delay period, in the control condition CA, participants were instructed to press and hold down a key as soon as a second, identical red square was displayed on screen. They then released the key when the second square disappeared, which occurred after a fixed duration of 400, 800, or 1000 msec. These durations were chosen to match the duration of the standard plus or minus an error, estimated on the basis of our previous findings using the same temporal tasks (i.e., overestimation of short and underestimation of long durations). To prevent anticipation, the duration of presentation of the second square was chosen randomly from the range described above, and independently from the duration of the first (standard) square. In the control condition $\mathrm{CP}$, the delay period was followed by the presentation of a second, identical red square lasting 400,800 , or 1000 msec. Participants were instructed to press a key as soon as the square disappeared. Thus, Tasks $\mathrm{A}$ and $\mathrm{P}$ are well matched to their respective control tasks CA and CP in terms of the visual stimulation and motor actions required. They differ from the control tasks in the requirement to keep and use timing information for reproduction or estimation of standard intervals, respectively.

\section{Paradigm}

Subjects performed each of the four tasks (A, P, CA, or $\mathrm{CP}$ ) in different blocks of trials in a blocked design for fMRI. Each scanning session comprised four runs of
10 blocks each. Each block comprised six trials from one of the four conditions. The duration of each block varied slightly, being determined by the subject's response (estimation/reproduction) in the two timing tasks and by the duration of the second square in the two control tasks. The longest block was, on average, $59.5 \mathrm{sec}$, and the shortest was $43.9 \mathrm{sec}$. Each block started with a brief presentation $(1.5 \mathrm{sec})$ of a key word that reminded the subject which task was to be performed.

In our previous work (Bueti \& Walsh, submitted), behavioral differences between $\mathrm{A}$ and $\mathrm{P}$ tasks were statistically more consistent at the shortest (300 msec) and at the longest $(1200 \mathrm{msec})$ durations. Therefore, in the present experiment, in which we were interested in the differences between the two tasks, stimuli with these durations made up two thirds of the trials in each block. The other third was represented by catch trials of 600 and $900 \mathrm{msec}$ durations, which served to better engage the subjects in the task. Therefore, in a block of six trials, a total of four different stimulus durations were represented. Two of these standard durations (300 and $1200 \mathrm{msec}$ ) were followed by a short delay and two were followed by a short and a long delay; two secondary durations (600 and $900 \mathrm{msec}$ ) were followed by either a long or a short delay. The order of presentation of each combination of durations and delays was randomized within the block; the order of the blocks was randomized and counterbalanced across runs and participants.

All participants were trained before scanning, performing the entire fMRI protocol in separate behavioral 
sessions. We tested 17 participants in total, but because the aim of the study was to find neural correlates of a behavioral/functional dissociation, only the 14 individuals who reliably underestimated or overestimated the standard duration after the long delay ( $8 \mathrm{sec}$ ) in the reproduction, but not in the estimation, task were taken forward to investigate with fMRI. Any generalization of the findings reported here to the population is constrained by this initial selection.

\section{fMRI Scanning}

A 1.5-T Siemens system was used to acquire $\mathrm{T} 2 *$-weighted echo-planar (EPI) image volumes with blood oxygenation level-dependent contrast. Each EPI volume comprised forty-eight $2-\mathrm{mm}$ axial slices with an in-plane resolution of $3 \times 3 \mathrm{~mm}$ positioned to cover the whole brain. Participants performed four runs, each consisting, on average, of 132 volumes (range 127-138). The first five volumes of each run were discarded to allow for T1 equilibration effects. Volumes were acquired continuously with a TR of $4.32 \mathrm{sec}$ per volume. In addition, a T1-weighted anatomical image was acquired for each participant.

\section{Analysis \\ Behavioral Data}

For $\mathrm{A}$ and $\mathrm{P}$ tasks the reproduced and the estimated times were recorded, respectively. Trials in which 600 and 900 msec were the standard durations were discarded; as explained earlier, these trials were considered "catch trials and dispersed unequally in the blocks. Mean accuracy was calculated as the difference between the reproduced (A) or the estimated (P) duration and the duration of the standard. A repeated measures analysis of variance was then performed on these difference values with the factors task (action, perception), duration $(300,1200 \mathrm{msec})$, and delay (1, $8 \mathrm{sec})$. All post hoc comparisons employed Tukey's HSD test with an alpha level of .05.

\section{fMRI Data}

Functional imaging data were analyzed using Statistical Parametric Mapping software (SPM2, Wellcome Department of Imaging Neuroscience, University College London). All image volumes were realigned, spatially normalized to a standard EPI template volume based on the MNI reference brain in the space of Talairach and Tournoux (1988), and resampled to $2 \mathrm{~mm}$ isotropic voxels. The normalized image volumes were then smoothed with an isotropic 9-mm full-width, half-maximum Gaussian kernel. These data were analyzed using a random effects model. Voxels that were activated in the experimental conditions were identified using a statistical model containing regressors that represented the responses evoked by the four tasks. Each experimental block (task) was modeled using a boxcar, convolved with a canonical hemodynamic response function. Each component of the model served as a regressor in a multiple regression analysis that in cluded the four experimental conditions (A, P, CA, CP) and the motion correction parameters (as effects of no interest). The data were high-pass filtered (cutoff frequency $=0.0083 \mathrm{~Hz}$ ) to remove low-frequency signal drifts, and global changes in activity were removed by proportional scaling. We initially performed 14 separate single-subject analyses, in which linear contrasts were used to test hypotheses about regionally specific condition effects. Statistical parametric maps of the $t$ statistic for each contrast of interest, transformed into corresponding $Z$ values, were defined for each subject.

The statistical contrasts used were the following:

1. $(\mathrm{A}-\mathrm{CA})$

2. $(\mathrm{P}-\mathrm{CP})$

3. $[(\mathrm{A}-\mathrm{CA})-(\mathrm{P}-\mathrm{CP})]$

4. $[(\mathrm{P}-\mathrm{CP})-(\mathrm{A}-\mathrm{CA})]$

The first two contrasts revealed changes in the brain activity reflecting the use of temporal information in either reproduction or estimation (after controlling for visual stimulation and motor action). The second two contrasts, representing the interaction of the two tasks (vs. their respective controls), were used to identify brain areas where activity was significantly greater when temporal information was used in the estimation (vs. reproduction) or vice-versa. These contrast maps were then entered into second-level random effects analyses, and statistical inferences for each contrast were derived using a one-sample $t$ test. A statistical threshold of $p<$ .05 , corrected at cluster level, was used, except for regions that were hypothesized a priori as part of the time network where a lower threshold of $p<.001$, uncorrected for multiple comparisons, was used. These areas were those consistently indicated in previous imaging studies as involved in time processing: basal ganglia, cerebellum, supplementary motor, premotor, prefrontal, and parietal cortex (Coull et al., 2004; Lewis, Wing, Pope, Praamstra, \& Miall, 2004; Macar, Anton, Bonnet, \& Vidal, 2004; Lewis \& Miall, 2003b; Nenadic et al., 2003; Rao et al., 2001; Schubotz et al., 2000; for review, see Macar et al., 2002).

\section{RESULTS}

\section{Behavioral Results}

Tasks were differently affected by the temporal delay $[$ Task $\times$ Duration $\times$ Delay effect: $F(1,13)=17,99, p<$ .001], as illustrated in Figure 2. The delay in task A, but not in task $\mathrm{P}$, biased performance significantly in favor of 


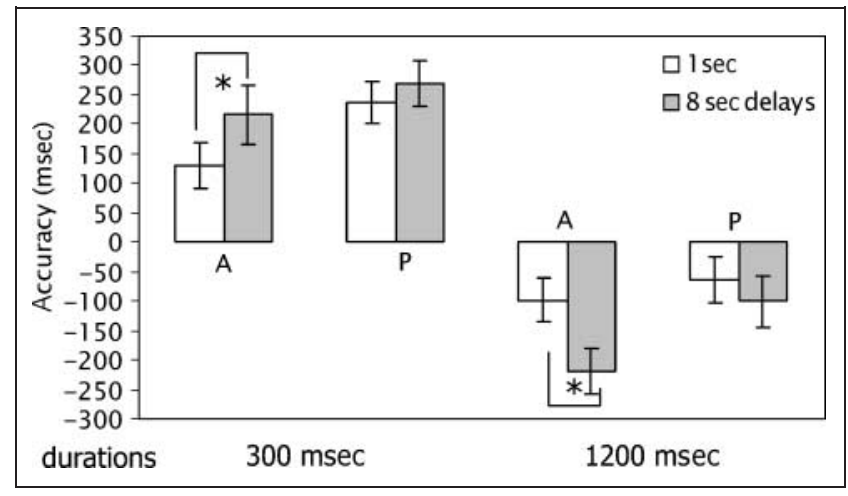

Figure 2. Behavioral results in tasks A and P for 14 subjects. Mean accuracy (msec; zero indicates perfect performance, positive values indicate overestimation, and negative values indicate underestimation of the standard duration) is plotted for the two main durations of the standard $(0.3$ and $1.2 \mathrm{sec})$. Bars show the accuracy in the two delay conditions for tasks $\mathrm{A}$ and $\mathrm{P}$ at the shortest and at the longest standard durations. It is apparent that the delay affected the performance only in task $\mathrm{A}$ at both durations.

overestimation of the short duration (300 msec, $p<.001$ ) and underestimation of the long one (1200 msec, $p<$ .001).

\section{fMRI Results}

$(A-C A)$

This contrast revealed areas whose activity was specifically associated with the reproduction of temporal information. Activated areas (see Table 1 for coordinates and $z$ values) included the right inferior parietal cortex (IPC), the left inferior frontal gyrus, the left middle frontal gyrus, and the left putamen (all significant at $p<.05$, corrected at cluster level; see Figure 3). Activations were also found in the bilateral SMA, right middle frontal gyrus, basal ganglia (right putamen and right globus pallidus), and cerebellum (left crus2, right VI lobule; for cerebellar taxonomy, we refer to Schmahmann et al., 1999).

$(P-C P)$

This contrast revealed areas whose activity was specifically associated with the use of temporal information in the estimation task. Activated areas (see Table 1 for coordinates and $z$ values, and Figure 3) included the left inferior frontal gyrus, left putamen, bilateral globus pallidum, right caudate nucleus, and right cerebellar hemisphere (right crus1), all significant at $p<.001$, uncorrected.

$$
[(A-C A)-(P-C P)]
$$

This contrast revealed areas where the reproduction task (vs. its control) evoked significantly more activity than the estimation task (vs. its control) (Figure 4). This revealed significant $(p<.05$, corrected) clusters of voxels located in the right IPC, in the left fusiform gyrus, in the cerebellar vermis, and in loci consistent with the extrastriate area V5 (Dumoulin et al., 2000). Activation was also observed in the right pre-SMA, left premotor cortex, left middle frontal gyrus, left IPC, and bilateral cerebellum (right VIII lobule, left crus2; all significant at $p<.001$, uncorrected for multiple comparison). Some of the areas activated in this contrast (fusiform gyrus bilaterally, left premotor cortex, left IPC) were not significant in none of the main effect separately $\mathrm{A}-\mathrm{CA}$ and $\mathrm{P}-\mathrm{CP}$. To rule out the possibility that those areas were inhibited in the contrast $\mathrm{P}-\mathrm{CP}$, perhaps because of the reactive response required only in task $\mathrm{CP}$ but not in $\mathrm{P}$, we run the $\mathrm{T}$ contrast $\mathrm{CP}-\mathrm{P}$. This contrast revealed significant activity in the cerebellum (right crus2: $12,-93,-33$, peak $z=4.12$ ), left angular gyrus $(-51,-66,39$, peak $z=3.95)$, right superior frontal gyrus $(15,30,60$, peak $z=3.70)$, left precuneus $(-12,-54,36$, peak $z=3.55)$, and right superior parietal cortex $(21,-69,60$, peak $z=3.50)$; all these locations were significant at $p<.05$, corrected at cluster level. No fusiform gyrus and left premotor cortex were significantly activated in this contrast. Activation in the left angular gyrus was more posterior than the location in the left IPC $(-36,-54,51)$ described in the interaction $(\mathrm{A}-\mathrm{CA})-(\mathrm{P}-\mathrm{CP})$. We could therefore conclude that the activations observed in the interactions were all correlated with the temporal task A.

$[(P-C P)-(A-C A)]$

This contrast indicated areas where the estimation task (vs. its control) evoked significantly more activity than the reproduction task (vs. its control). However, there were no suprathreshold clusters for this statistical comparison.

\section{DISCUSSION}

Here we investigated the neural substrate for processing time for both reproduction and estimation. We employed closely matched time reproduction (task A) and time estimation tasks (task P) that allowed a direct comparison in the same participants between different ways of using temporal information. This direct comparison has not previously been explored in the same fMRI experiment. Using the same set of visual stimuli and the same millisecond range of durations, we identified commonalities and differences in brain areas involved in the reproduction and the estimation of temporal intervals.

Behavioral performance differed between the two tasks. Specifically, the presence of a long delay (8 sec) 
Table 1. Stereotaxic Brain MNI Coordinates $(\mathrm{mm})$ for Regions Activated in the Different Contrasts at $p<.001$, Uncorrected for Multiple Comparisons and $p<.05$ Corrected at Cluster Level

$\begin{array}{lllll}x & y & \text { Z-score } & \text { Voxels }(n)\end{array}$

$A-C A$

Frontal cortex

Inferior frontal gyrus pars triangularis (BA 45) L

$-48$

Middle frontal gyrus (DLPC, BA 46) L*

Middle frontal gyrus (DLPFC, BA 46) R

SMA R

SMA L

Parietal cortex

Supramarginal gyrus (BA 40) $\mathrm{R}^{*}$

Basal ganglia

Putamen L*

Putamen R

Globus pallidus R

Cerebellum

Crus2 L

VI R

$P-C P$

Frontal cortex

Inferior frontal gyrus pars opercularis (BA 44) L

Basal ganglia

Putamen L

Globus pallidus R

Nucleus caudatus $R$

Cerebellum

Crus1 R

$(A-C A)-(P-C P)$

Frontal cortex

Middle frontal gyrus (DLPFC, BA 46) L

Pre-SMA R

Premotor L

Parietal cortex

Supramarginal gyrus (BA 40) R*

Inferior parietal L

Temporal cortex

Inferior temporal cortex (V5/MT)*

Fusiform gyrus L*

Fusiform gyrus R

Cerebellum

Vermis*
VIII R
Crus2 L

$-57$

$-27$

21

27

42

24

12

24

3.14

35

$-60$

$-36$

3.6

13

$\begin{array}{rr}-48 & 27 \\ 12 & 24 \\ -45 & 12\end{array}$

39

60

51

3.77

3.44

3.03

98

35

$\begin{array}{rrrrr}48 & -39 & 45 & 4.86 & 235 \\ -36 & -54 & 51 & 3.23 & 45\end{array}$

$54-66$

$-30 \quad-63$

$-66$

$-9$

$-9$

$-9$

4.01

301

198

14

\begin{tabular}{rrrrr}
0 & -78 & -24 & 3.71 & 145 \\
12 & -63 & -36 & 3.49 & 86 \\
-30 & -66 & -45 & 3.41 & 52 \\
\hline
\end{tabular}

$\mathrm{BA}=$ Brodmann's area; $\mathrm{DLPFC}=$ dorsolateral prefrontal cortex; $\mathrm{SMA}=$ supplementary motor area; $\mathrm{L}=$ left $\mathrm{R}=$ right; $\mathrm{A}=\mathrm{action} ; \mathrm{P}=$ perception; $\mathrm{CA}=$ control for action; $\mathrm{CP}=$ control for perception.

*Significant at $p<.05$, corrected at cluster level. 
between stimulus presentation and response biased performance in the reproduction but not in the estimation task. This replicates our earlier behavioral findings (Bueti \& Walsh, submitted) and indicates that, akin to spatial information, time is processed differently depending on what purpose the information will serve (Bradshaw \& Watt, 2002). We are now able to identify the neural substrate of these two processes. Strikingly, although the two tasks activated common areas previously associated with the use of timing information (the basal ganglia and the cerebellum), there were also strong differences between tasks in the activation of a number of different cortical regions. Specifically, in the reproduction task where time information was used for reproduction, a number of different cortical areas were differentially activated, including the right SMA, left middle frontal gyrus, and left premotor cortex. Moreover, such differential activation was particularly reliable in the right inferior parietal cortex, left fusiform gyrus, and in the right extrastriate visual area V5/MT. This latter area, in particular, has not previously been identified in timing-related research, but its involvement has been predicted on theoretical grounds (Walsh, 2003) and has been anticipated by recent psychophysical work (Johnston, Arnold, \& Nishida, 2006; Kanai \& Watanabe, 2006).

\section{Areas Commonly Active for Both Reproduction and Estimation of Time}

The basal ganglia (bilateral putamen and globus pallidus) were activated in both tasks (vs. matched control tasks). This corroborates findings from patients with Parkinson's disease that dopaminergic treatment improves both motor timing (O'Boyle et al., 1996; Pastor, Jahanshahi, Artieda, \& Obeso, 1992) and time perception (Malapani et al., 1998). Pharmacological manipulations in animals also suggest that dopaminergic antagonist and agonists respectively slow-down and speed-up timing operations (Matell et al., 2003; Maricq \& Church, 1983). The striatal hypothesis has been supported also by neuroimaging studies that have documented activity in the striatum during temporal tasks

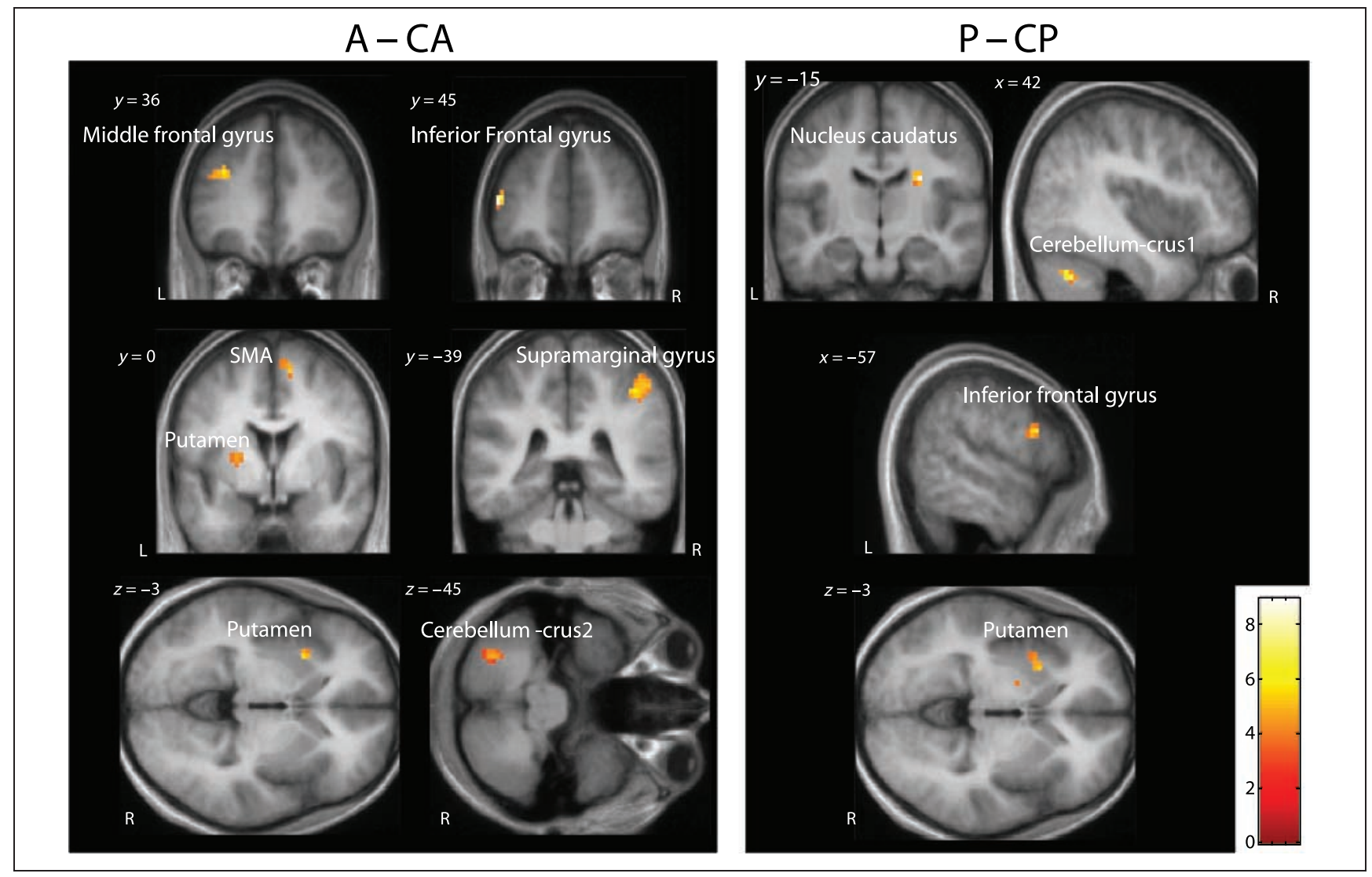

Figure 3. Loci activated by different statistical contrasts (see Results) overlaid on the average T1-weighted structural image from the 14 subjects in the stereotactic space of Talairach and Tournoux (1988). A threshold of $T=3.85$ is used for display purposes. Loci activated in contrast A - CA reveals areas active in the time reproduction task compared to its control. Activation is apparent in the right supramarginal gyrus (SMG), left middle frontal cortex, left inferior frontal cortex, left putamen (all significant $p<.05$, corrected at cluster level), right putamen, right globus pallidus, bilateral cerebellum (left crus2, right VI lobule, bilateral SMA; all significant $p<.001$, uncorrected). Loci activated in contrast $\mathrm{P}-\mathrm{CP}$ reveals areas active in the estimation task (P) compared to its control. Activations were observed in the left inferior frontal cortex, left putamen, right caudate nucleus, right globus pallidus, right cerebellum (crus1) (all significant $p>.001$, uncorrected). 
Figure 4. Loci activated by the statistical contrast $(\mathrm{A}-\mathrm{CA})-(\mathrm{P}-\mathrm{CP})$ (see Results) overlaid on the average T1-weighted structural image from the 14 subjects in the stereotactic space of Talairach and Tournoux (1988). This contrast shows brain areas where the reproduction task (vs. its control) evoked significantly more activity than the estimation task (vs. its control). Activations were found in the right supramarginal gyrus (SMG), right V5/MT, left fusiform gyrus, cerebellar vermis (all significant $p<.05$, corrected at cluster level), left middle frontal gyrus, right pre-SMA, left premotor cortex, left SMG, bilateral cerebellum (left crus2 and right VIII lobule, all significant $p<$ .001, uncorrected)

\section{$(A-C A)-(P-C P)$}

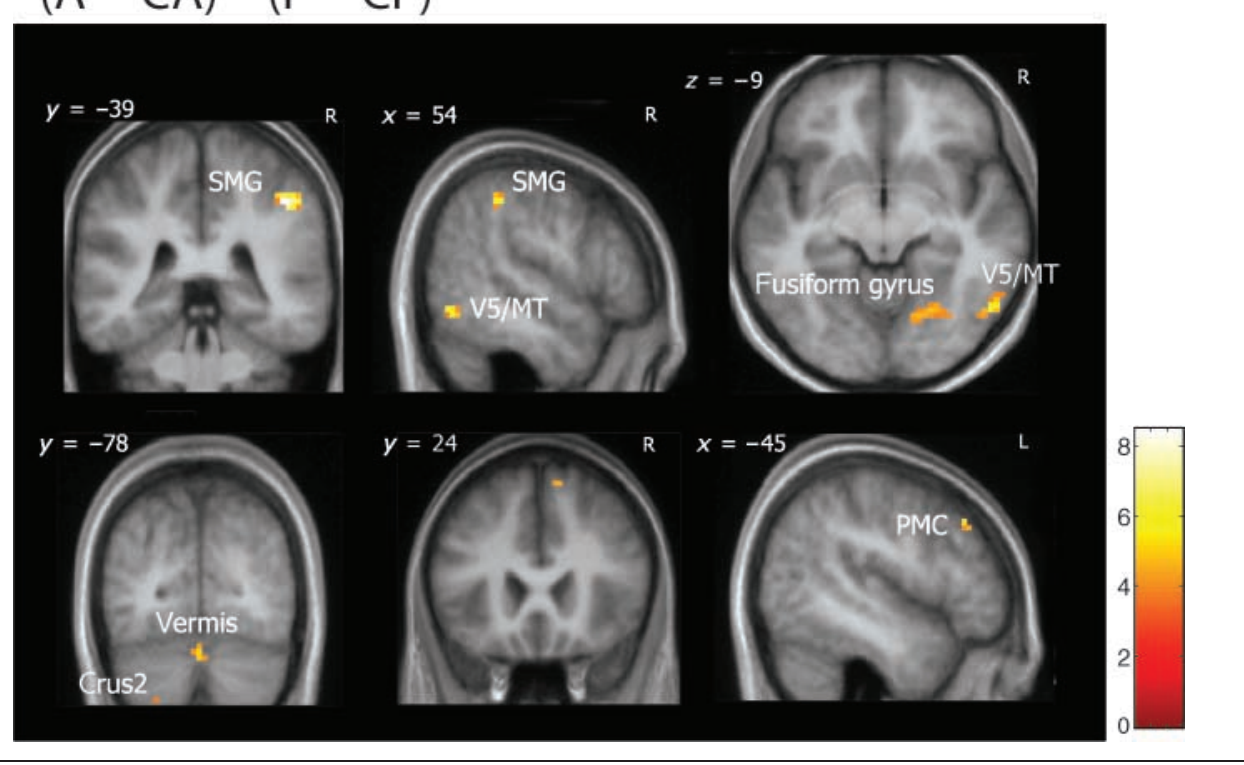

(Coull et al., 2004; Nenadic et al., 2003) especially in the early stage (encoding) of time processing (Rao et al., 2001).

We also found cerebellar activity in both action and perception tasks, although activation of the vermis was prominent only in the reproduction task. We hypothesize that the activity observed in the lateral cerebellum reflects timekeeping operations common to both tasks. Indeed, it is well established that the lateral, but not medial, cerebellum is involved in timekeeping operations (Ivry et al., 1988). Furthermore, activation of the lateral cerebellar hemispheres, rather than the medial part, has previously been observed in timing-related fMRI studies (Lewis et al., 2004; Macar et al., 2004; Schubotz et al., 2000; Rao et al., 1997). These findings generally support the hypothesis that temporal perception and production are sustained by the beat of the same clock, and that the dopaminergic neurotransmission of the striatum and the activity of the lateral cerebellum are likely to be implicated in these hypothetical internal timekeeping mechanisms. The activity in the vermis found only in the reproduction task may be more related to the multisensory integrative processes that were only necessary in the reproduction task, where both visual (the square on the screen) and proprioceptive (pressing and holding down the key) information had to be accurately integrated to reproduce the standard duration. It is well known that the cerebellar vermis and the adjacent intermediate part of the cerebellar hemispheres receive auditory, visual, vestibular, and somatic inputs (Kandel, Schwartz, \& Jessell, 2000, fourth edition).

\section{Areas Differentially Active in Reproduction and Estimation of Time}

The two timing tasks we employed shared identical sensory components (the same visual stimuli with an identical range of durations), memory, and attentional demands. Moreover, in both tasks, there was a form of temporal planning (i.e., prepare to release the key in task A vs. prepare to press the key in task P). Any asymmetries in motor components were matched by the appropriate control tasks. Areas more active in the reproduction task (vs. its control) compared to the estimation task (vs. its control) therefore reflected a difference in how temporal information is coded, using a motor or a perceptual representation. Such areas included the left middle frontal gyrus, the left premotor cortex, the right pre-SMA and, more reliably, the IPC (especially in the right hemisphere), the left fusiform gyrus, and a region of the extrastriate cortex consistent with the stereotactic location of V5/MT. Involvement of the IPC in time perception is well established. Specifically, right IPC activity has been observed in neuroimaging (Lewis \& Miall, 2003a, 2003b; Rao et al., 2001) and electrophysiological experiments (Mohl \& Pfurtscheller, 1991), investigating the role of this area in temporal processing. Moreover, patients with right IPC damage have substantial time-related perception deficits (Harrington, Haaland, \& Knight, 1998) and repetitive transcranial magnetic stimulation (rTMS) of the right IPC disrupts performance on prospective time judgments (Alexander, Cowey, \& Walsh, 2005; Bueti, Bahrami, \& Walsh, in press). However, the precise functional role of 
the parietal cortex in time perception has been investigated rather less to date than those of subcortical structures. As a consequence, cortical processes have been loosely associated with all stages of temporal processing. Most studies emphasize the attentional role of the parietal cortex in timing functions (Lewis \& Miall, 2003a; Coull \& Nobre, 1998). However, our new findings cannot be explained by this hypothesis, as the attentional load and the sensory components were identical in both reproduction and estimation tasks. Instead, we suggest that the parietal cortex plays a role as the interface between sensory and motor processes. The involvement of the parietal cortex in time processing for reproduction that we demonstrate here allows an interesting parallel with the representation of space and time. Because the parietal cortex plays a role in the "where" and "how" of spatial vision (Milner \& Goodale, 1995), it may also use temporal information to ensure that the metrics employed in space are employed at the right moment, which requires accurate spatio-temporal integration. In terms of temporal processing, we suggest that the parietal cortex plays a role mediating between the central clock and peripheral motor effectors. Although we found stronger activation in the right IPC, the left IPC was also differentially activated in the interaction between reproduction and estimation tasks (vs. their respective controls). We propose that the right IPC uses representational systems, perhaps spatially encoded, which are common to time space and quantity as relevant to action and this representation is available to left hemisphere areas required for action selection and generation (Walsh, 2003; Rushworth, Ellison, \& Walsh, 2001).

The activation observed in the left middle frontal gyrus may be explained in the context of the strong parietal activation. The prefrontal and posterior parietal cortex are well known to be anatomically and functionally interconnected, and have been implicated in preparation of behavioral action (Quintana \& Fuster 1999; Andersen, 1987). Prefrontal activity may be temporally specific, indeed the firing rate of neurons in the prefrontal cortex has been found to be time modulated if temporal information was relevant to guide a saccade (Genovesio, Tsujimoto, \& Wise, 2006).

We also found differential involvement of V5/MT in the reproduction task (vs. its control) compared to the estimation task (vs. its control). Such a new finding was anticipated by previous TMS study (Bueti, Bahrami, \& Walsh, in press). Specifically, rTMS over V5/MT significantly impairs discrimination of visual, but not auditory, time intervals. The TMS data have been interpreted in terms of existence of visual local time mechanisms, according to the notion of distributed timers (Mauk \& Buonomano, 2004; Buonomano \& Merzenich, 1995). The importance of local visual time mechanisms has been recently advanced by studies showing that the apparent duration of a visual stimulus can be spatially manipulated by adapting to a fast motion or flicker (Johnston et al., 2006) and that a proportion of neurons in the primary visual cortex of rats fire in a way that seems to predict the timing of the reward (Shuler \& Bear, 2006).

The imaging data presented here help to specify the role of V5/MT in time processing by showing that this area is involved not just in the discrimination (as demonstrated in TMS experiments) but also in the reproduction of visual time intervals. Activity in V5/MT was observed only in the action task (vs. its control) compared to the estimation task (vs. its control). This may be due to the visual spatial role of this area. V5/MT is a motion sensitive area and perception of visual motion is linked to making predictions and anticipation of spatial positions. Indeed, adaptation to motion changes subsequent perception of spatial localization (McGraw, Whitaker, Skillen, \& Chung, 2002). Making predictions and anticipation of spatial position is particularly relevant for actions, where presumably we need accurate processing of spatial as well as temporal information.

Activations of the left premotor cortex and the right SMA, although less statistically robust than those of the right IPC and right V5/MT, were also found. These activations might be correlated with the stronger timed motor components of task A compared to task P. These activations might be temporally specific as previous studies show involvement of these areas in time processing. For example, activations in the premotor and SMA are found in the human brain during the reproduction of different rhythms in finger tapping tasks (Lewis et al., 2004; Rao et al., 1997).

As specified above, Tasks $\mathrm{A}$ and $\mathrm{P}$ are not completely dissociated in their motor and perceptual components; both tasks required a form of timed motor preparation (i.e., prepare to press and release the key in task A vs. prepare only to press the key in task $\mathrm{P}$ ). However, only in task A are temporal judgments entirely based on a motor representation. Our functional and behavioral results have to be, therefore, interpreted considering the limits of the distinction between these tasks.

\section{Conclusion}

Here, we investigated the neural correlates of the use of temporal information for either reproduction or estimation. Behaviorally, we confirmed dissociations in performance comparing the two tasks. Both tasks were associated with activation of distributed networks of cortical and subcortical brain regions, but there were also important differences between tasks. Activation of the basal ganglia and the cerebellum was common to both tasks, whereas a different and a wider cortical network was differentially activated during the reproduction of time compared to just estimation. This network included the right pre-SMA, the left middle frontal gyrus, the left premotor cortex, the fusiform gyrus, and specific involvement of the inferior parietal cortices and the visual extrastriate area V5/MT. 
In addition to finding commonalities between the tasks which, to some extent, support the idea of a specialized time network (a core of time), our finding also suggests that the nature of the timing task may be critical to which brain areas are recruited.

\section{Acknowledgments}

This work was supported by the Wellcome Trust (G. R.), Leverhulme Trust (D. B.), and the Royal Society (V. W.).

Reprint requests should be sent to Domenica Bueti, Institute of Cognitive Neuroscience, University College London, 17 Queen Square, London WC1N 3AR, UK, or via e-mail: d.bueti@ucl.ac.uk.

\section{REFERENCES}

Alexander, I., Cowey, A., \& Walsh, V. (2005). The right parietal cortex and time perception: Back to Critchley and the Zeitraffer phenomenon. Cognitive Neuropsychology, 22, 306-315.

Andersen, R. A. (1987). The role of the inferior parietal lobule in spatial perception and visual-motor integration. In F. Plum, V. B. Mountcastle, \& S. R. Geiger (Eds.), The bandbook of physiology, Section 1: The nervous system, Vol. V: Higher functions of the brain. Bethesda, MD: American Physiological Society.

Artieda, J., Pastor, M. A., Lacruz, F., \& Obeso, J. A. (1992). Temporal discrimination is abnormal in Parkinson's disease. Brain, 115, 199-210.

Bueti, D., Bahrami, B., \& Walsh, V. (in press). Sensory and association cortex in time perception. Journal of Cognitive Neuroscience.

Bueti, D., \& Walsh, V. (submitted). Memory for time distinguishes between perception and action.

Buonomano, D. V., \& Merzenich, M. M. (1995). Temporal information transformed into a spatial code by a neural network with realistic properties. Science, 267, 1028-1030.

Bradshaw, M. F., \& Watt, S. J. (2002). A dissociation of perception and action in normal human observers: The effect of temporal-delay. Neuropsychologia, 40, $1766-1778$

Clarke, S., Ivry, R., Grinband, J., \& Shimizu, N. (1996). In G. E. S. Vroon \& P. A. Vroon (Eds.), Time, internal clocks, and movement (pp. 257-280). New York: Elsevier.

Coull, J. T., \& Nobre, A. C. (1998). Where and when to pay attention: The neural systems for directing attention to spatial locations and to time intervals as revealed by both PET and fMRI. Journal of Neuroscience, 18, 7426-7435.

Coull, J. T., Vidal, F., Nazarian, B., \& Macar, F. (2004). Functional anatomy of the attentional modulation of time estimation. Science, 303, 1506-1508.

Dumoulin, S. O., Bittar, R. G., Kabani, N. J., Baker, C. L., Jr., Le Goualher, G., Bruce Pike, G., et al. (2000). A new anatomical landmark for reliable identification of human area V5/MT: A quantitative analysis of sulcal patterning. Cerebral Cortex, 10, 454-463.

Genovesio, A., Tsujimoto, S., \& Wise, S. P. (2006). Neural activity related to elapsed time in prefrontal cortex. Journal of Neurophysiology, 95, 3281-3285.

Gibbon, J., Malapani, C., Dale, C. L., \& Gallistel, C. (1997). Toward a neurobiology of temporal cognition: Advances and challenges. Current Opinion in Neurobiology, 7 , 170-184.
Harrington, D. L., Haaland, K. Y., \& Hermanowicz, N. (1998). Temporal processing in the basal ganglia. Neuropsychology, $12,3-12$

Harrington, D. L., Haaland, K. Y., \& Knight, R. T. (1998). Cortical networks underlying mechanisms of time perception. Journal of Neuroscience, 18, 1085-1095.

Ivry, R., \& Keele, S. (1989). Timing functions of the cerebellum. Journal of Cognitive Neuroscience, 1, 136-152.

Ivry, R., Keele, S., \& Diener, H. C. (1988). Dissociation of the lateral and medial cerebellum in movement timing and movement execution. Experimental Brain Research, 73, 167-180.

Ivry, R. B., \& Hazeltine, R. E. (1995). The perception and production of temporal intervals across a range of durations: Evidence for a common timing mechanism. Journal of Experimental Psychology: Human Perception and Performance, 21, 1-12.

Johnston, A., Arnold, D. H., \& Nishida, S. (2006). Spatially localized distortions of event time. Current Biology, 16, 472-479.

Kanai, R., \& Watanabe, M. (2006). Visual onset expands subjective time. Perception and Psychophysics, $68,1113-1123$.

Kandel, E. R., Schwartz, J. H., \& Jessell, T. M. (2000). Principles of neural science (4th ed.). New York: McGraw-Hill.

Keele, S. W., Pokorny, R. A., Corcos, D. M., \& Ivry, R. (1985). Do perception and motor production share common timing mechanisms: A correlational analysis. Acta Psychologica, 60, 173-191.

Lewis, P. A., \& Miall, R. C. (2003a). Distinct systems for automatic and cognitively controlled time measurement: Evidence from neuroimaging. Current Opinion in Neurobiology, 13, 250-255.

Lewis, P. A., \& Miall, R. C. (2003b). Brain activation patterns during measurement of sub- and supra-second intervals. Neuropsychologia, 41, 1583-1592.

Lewis, P. A., Wing, A. M., Pope, P. A., Praamstra, P., \& Miall, R. C. (2004). Brain activity correlates differentially with increasing temporal complexity of rhythms during initialisation, synchronisation, and continuation phases of paced finger tapping. Neuropsychologia, 42, 1301-1312.

Macar, F., Anton, J. L., Bonnet, M., \& Vidal, F. (2004). Timing functions of the supplementary motor area: An event-related fMRI study. Cognitive Brain Research, 21, 206-215.

Macar, F., Lejeune, H., Bonnet, M., Ferrara, A., Pouthas, V., Vidal, F., et al. (2002). Activation of the supplementary motor area and of attentional networks during temporal processing. Experimental Brain Research, 142, 475-485.

Malapani, C., Rakitin, B., Levy, R., Meck, W. H., Deweer, B., Dubois, B., et al. (1998). Coupled temporal memories in Parkinson's disease: A dopamine-related dysfunction. Journal of Cognitive Neuroscience, 10, 316-331.

Maricq, A. V., \& Church, R. M. (1983). The differential effects of haloperidol and methamphetamine on time estimation in the rat. Psychopharmacology, 1, 10-15.

Matell, M. S., Meck, W. H., \& Nicolelis, M. A. (2003). Interval timing and the encoding of signal duration by ensembles of cortical and striatal neurons. Behavioral Neuroscience, 117, 760-773.

Mauk, M. D., \& Buonomano, D. V. (2004). The neural basis of temporal processing. Annual Review of Neuroscience, 27, 307-330.

McGraw, P. V., Whitaker, D., Skillen, J., \& Chung, S. T. (2002). Motion adaptation distorts perceived visual position. Current Biology, 12, 2042-2047.

Milner, D., \& Goodale, M. (1995). The visual brain in action. Oxford: Oxford University Press. 
Mohl, W., \& Pfurtscheller, G. (1991). The role of the right parietal region in a movement time estimation task. NeuroReport, 2, 309-312.

Nenadic, I., Gaser, C., Volz, H. P., Rammsayer, T., Hager, F., \& Sauer, H. (2003). Processing of temporal information and the basal ganglia: New evidence from fMRI. Experimental Brain Research, 148, 238-246.

O'Boyle, D. J., Freeman, J. S., \& Cody, F. W. (1996). The accuracy and precision of timing of self-paced, repetitive movements in subjects with Parkinson's disease. Brain, 119, 51-70.

Pastor, M. A., Jahanshahi, M., Artieda, J., \& Obeso, J. A. (1992). Performance of repetitive wrist movements in Parkinson's disease. Brain, 115, 875-891.

Quintana, J., \& Fuster, J. M. (1999). From perception to action: Temporal integrative functions of prefrontal and parietal neurons. Cerebral Cortex, 9, 213-221.

Rammsayer, T. H. (1999). Neuropharmacological evidence for different timing mechanisms in humans. Quarterly Journal of Experimental Psychology: Series B, 52, $273-286$.

Rao, S. M., Harrington, D. L., Haaland, K. Y., Bobholz, J. A., Cox, R. W., \& Binder, J. R. (1997). Distributed neural systems underlying the timing of movements. Journal of Neuroscience, 17, 5528-5535.

Rao, S. M., Mayer, A. R., \& Harrington, D. L. (2001). The evolution of brain activation during temporal processing. Nature Neuroscience, 4, 317-323.

Rushworth, M. F., Ellison, A., \& Walsh, V. (2001). Complementary localization and lateralization of orienting and motor attention. Nature Neuroscience, 4, 656-661.
Schmahmann, J. D., Doyon, J., McDonald, D., Holmes, C., Lavoie, K., Hurwitz, A. S., et al. (1999). Three-dimensional MRI atlas of the human cerebellum in proportional stereotaxic space. Neuroimage, 10, 233-260.

Schubotz, R., \& Friederici, A. D. (1997). Electrophysiological correlates of temporal and spatial information processing. NeuroReport, 8, 1981-1986.

Schubotz, R. I., Friederici, A. D., \& von Cramon, D. Y. (2000). Time perception and motor timing: A common cortical and subcortical basis revealed by fMRI. Neuroimage, $11,1-12$.

Shuler, M. G., \& Bear, M. F. (2006). Reward timing the primary visual cortex. Science, 311, 1606-1609.

Spencer, R. M., Zelaznik, H. N., Diedrichsen, J., \& Ivry, R. B. (2003). Disrupted timing of discontinuous but not continuous movements by cerebellar lesions. Science, 300, 1437-1439.

Talairach, J., \& Tournoux, P. (1988). Co-planar stereotaxic atlas of the buman brain. New York: Thieme.

Treisman, M., Faulkner, A., \& Naish, P. L (1992). On the relation between time perception and timing of motor action: Evidence for a temporal oscillator controlling the timing of movement. Quarterly Journal of Experimental Psychology, 45, 235-263.

Treisman, M., Faulkner, A., Naish, P. L., \& Brogan, D. (1990). The internal clock: Evidence for a temporal oscillator underlying time perception with some estimates of its characteristic frequency. Perception, 6, 705-743.

Walsh, V. (2003). A theory of magnitude: Common cortical metrics of time, space and quantity. Trends in Cognitive Sciences, 7, 483-488. 